\section{Hoppe-Seyler, Felix}

H. Fiedler

Erfurt, Deutschland

Lebensdaten Felix (auch Ernst Felix Immanuel) HoppeSeyler war deutscher Arzt und Physiologe, geboren 1825 in Freyburg (Unstrut), gestorben 1895 in Wasserburg (Bodensee). Sein Schwager Pastor Georg Seyler adoptierte ihn nach dem Tod seiner Eltern und gab ihm den Doppelnamen. Sein Enkel Felix Adolf Hoppe-Seyler (1898-1945) leitete die Physiologisch-Chemischen Institute in Würzburg (1930-1934) und Greifswald (1934-1945).

Hoppe-Seyler studierte Medizin in Halle, Leipzig, Prag und Wien und promovierte 1850 in Berlin. Nach 2 Jahren als praktischer Arzt wurde er 1854 Prosektor im Institut für Anatomie und Physiologie in Greifswald. 1856 wechselte er in die Chemische Abteilung des Virchowschen Pathologischen Instituts und wurde 1861 als Professor für Angewandte Chemie nach Tübingen berufen. In einem primitiven Labor in der umgebauten Küche des Schlosses Hohentübingen wurden so viele wichtige Ergebnisse erbracht, dass 2015 der Raum als Museum der Tübinger Biochemie eingeweiht wurde. 1872-1895 war er Professor für Physiologische Chemie in Straßburg. Die \ Deutsche Gesellschaft für Klinische Chemie und Laboratoriumsmedizin e.V. (DGKL) vergibt seit 1985 alle 3 Jahre den Felix-Hoppe-Seyler-Preis für besondere wissenschaftliche Leistungen.

Verdienste Hoppe-Seyler gilt als Begründer der Physiologische Chemie und hat deren Bedeutung in Lehre und Klinik immer wieder betont und durchgesetzt. An allen Wirkungs- stätten hat er eine große Zahl von Studenten und Ärzten aus vielen Ländern angeleitet, promoviert und habilitiert (wenige Beispiele): Alexander Schmidt (Blutgerinnung, Fibrinogen), Ernst von Leyden, Wilhelm Kühne (Enzyme), Friedrich Miescher (1844-1895, Entdeckung des „Nuclein“), Eugen Baumann (Schwefelstoffwechsel, Homogentisinsäure), Ernst Leopold Salkowski (Phenolausscheidung, Autolyse, Pentosurie), Albrecht, Kossel, Rudolf von Jaksch (Acetoacetat, Melanintest), Josef von Mering (entdeckte mit O. Minkowski durch Pankreatektomie die Ursache des Typ-1-Diabetes).

Hoppe-Seyler forschte über Blutfarbstoffe und Chlorophyll. Er bewies, dass Hämoglobin eine definierte chemische Substanz ist und Eisen enthält. Mit einem verbesserten Spektroskop fand er verschiedene Absorptionsbanden und deren Veränderung durch Sauerstoffaufnahme und Bildung von $\mathrm{CO}-\mathrm{Hb}$ und Met-Hb. Er bewies, dass die Oxidation zur Energiegewinnung nicht im Blut, sondern in der Zelle erfolgt. Mit seinen Schülern untersuchte er die Zusammensetzung der Zellen (Nukleoproteine, Lezithin, Albumin, Cholesterin und Glykosamine) und analysierte Knorpel, Knochen und Zahnschmelz. 1877 gründete er die Zeitschrift für Physiologische Chemie.

\section{Literatur}

Hoppe-Seyler F (1858) Handbuch der physiologisch und pathologischchemischen Analyse, 8. Aufl. 1909. Hirschwald, Berlin Hoppe-Seyler F (1877-1881) Textbuch der Physiologischen Chemie, 4 Bde. Hirschwald, Berlin

Mathews AP (1898) The life and work of Felix Hoppe-Seyler. Popular Science Monthly 53:August. https://en.wikisource.org/wiki/Popu lar_Science_Monthly/Volume_53/August1898. Zugegriffen im Sept. 2016 